EPJ Web of Conferences 32, 01016 (2012)

DOI: $10.1051 /$ epjconf/20123201016

(C) Owned by the authors, published by EDP Sciences, 2012

\title{
Effect of density fluctuations on ECCD in ITER and TCV
}

\author{
J. Decker ${ }^{1, a}$, Y. Peysson ${ }^{1}$, and S. Coda ${ }^{2}$ \\ 1 CEA, IRFM, F-13108, Saint-Paul-lez-Durance, France. \\ 2 EPFL, CRPP, Association EURATOM - Confédération Suisse, CH - 1015 Lausanne, Switzerland.
}

\begin{abstract}
Density fluctuations near the edge of tokamak plasmas can affect the propagation of electron cyclotron (EC) waves. In the present paper, the EC wave propagation in a fluctuating equilibrium is determined using the ray-tracing code $\mathrm{C} 3 \mathrm{PO}$. The evolution of the electron distribution function is calculated self-consistently with the EC wave damping using the 3-D Fokker-Planck solver LUKE. The cumulative effect of fluctuations results in a significant broadening of the current profile combined with a fluctuating power deposition profile. This mechanism improves the simulation of fully non-inductive EC discharges in the TCV tokamaks. Predictive simulations for ITER show that density fluctuations could make the stabilization of NTMs in ITER more challenging.
\end{abstract}

\section{Introduction}

Electron cyclotron current drive (ECCD) systems are characterized by the possibility to drive highly localized currents in a predictable and controlled manner. ECCD is a primary choice for current profile control in present day tokamaks such as the tokamak à configuration variable (TCV) [1], and a $20 \mathrm{MW}$, $170 \mathrm{GHz}$ ECCD system is planned for the control of neoclassical tearing modes (NTMs) in ITER [2].

Large levels of electron density fluctuations are often measured near the plasma edge and could affect the propagation and thus the damping of EC waves. In the TCV tokamak, conventional raytracing/Fokker-Planck simulations of fully non-inductive discharges tend to overestimate the driven current by up to a factor two [3-5]. While the introduction of large ad-hoc fast electrons radial transport in simulations reduces the driven current, the corresponding reconstructed bremsstrahlung emission is well below experimental measurements from the hard X-ray diagnostic $[4,5]$. By broadening the current profile without transporting fast electrons, density fluctuations could provide a better mechanism to improve the simulations. In ITER, first-harmonic heating combined with high pedestal density in some scenarios yield high values of the susceptibility tensor elements and a potentially large effect of density fluctuations on the EC wave propagation. In addition, the relatively long distance from the edge to the current deposition region means that even small perturbations in the wave propagation could lead to a significant displacement or broadening of the current profile considering the requirements for NTM stabilization [2].

The effect of fluctuations on the propagation and damping of radio-frequency waves has been investigated previously using a scattering formalism, both for lower-hybrid waves [6-8] and, more recently, for EC waves in the presence of blobs [9-11]. In the present paper, the effect of density fluctuations on ECCD is described using a new method [12], in which the time-dependent wave propagation is calculated using the ray-tracing code $\mathrm{C} 3 \mathrm{PO}$ [13] in a fluctuating plasma equilibrium. The time evolution of the electron distribution function is calculated self-consistently using the 3-D FokkerPlanck code LUKE [14]. The effect of fluctuations is determined from a statistical analysis of ECCD calculated at successive times in the fluctuating equilibrium. The results are found to be somewhat similar to those obtained from a scattering formalism [12].

\footnotetext{
a e-mail: joan.decker@cea.fr
} 
A density fluctuations model derived from drift-wave turbulence [12] is presented in Section 2. The calculation of ECCD in a fluctuating equilibrium is described in Section 3. The driven current and the bremsstrahlung emission, calculated by the code R5X2 [15], are moments of the distribution function that can be compared to experimental observations of fully non-inductive EC discharges in the TCV tokamak (Section 4). In ITER, the broadening of the current profile and the fluctuating power deposition are compared to requirements for NTM stabilization (Section 5).

\section{Modeling of density fluctuations}

The effect of fluctuations on ECCD is calculated assuming a constant background equilibrium characterized by an axisymmetric magnetic field configuration $\mathbf{B}(\rho, \theta)$ of arbitrary shape, and some electron density $\bar{n}_{e}(\rho)$ and temperature profiles $\bar{T}_{e}(\rho)$. The normalized radius $\rho$ is defined as the square root of the normalized toroidal flux, and $\theta$ is the poloidal angle. Electron density fluctuations are described by an arbitrary space- and time-dependent function $\tilde{n}_{e}(\rho, \theta, \phi)$, which can be derived from analytical or numerical models, experimental measurements, etc. In this paper, a model based on drift-wave turbulence characterized by a Gaussian 1-D spacial autocorrelation function with amplitude $\sigma_{f}$ and correlation length $\xi_{\perp}$ yields a superposition of independent modes of the form [12]

$$
\frac{\tilde{n}_{e}}{\bar{n}_{e}}=F_{\lambda} F_{\Delta}\left[2 \sigma_{f} \pi^{1 / 4} \sqrt{\frac{\xi_{\perp}}{L_{\perp}}} \sum_{p=1}^{p_{\max }} \exp \left(-\frac{\pi^{2}}{2} p^{2} \frac{\xi_{\perp}^{2}}{L_{\perp}^{2}}\right) \sin \Phi_{p}\right]
$$

where $\Phi_{p}=p \vartheta+2 \pi \chi_{p}$ is the phase for the Fourier mode $p$ and $\chi_{p}$ is a random number between 0 and 1 . The function $F_{\lambda}=[1+\lambda+(1-\lambda) \cos \theta] / 2$ accounts for ballooning effects on the fluctuation amplitude. The localization of fluctuations near the plasma edge is described by the function $F_{\Delta}=$ $\exp \left[-\ln 2(\rho-1)^{2} / \Delta^{2}\right]$ parametrized by the half width at half-maximum $\Delta$. The angle $\vartheta(\rho, \theta, \phi)$ defined by $\mathbf{B} \cdot \nabla \vartheta=0$ and $\nabla \rho \cdot \nabla \vartheta=0$ parametrizes the direction perpendicular to the magnetic field within flux surfaces, and defines the drift wave structure. The reference length $L_{\perp}$ represents approximately the poloidal path of a field line over one toroidal period.

\section{ECCD calculations in the presence of density fluctuations}

\subsection{Space scales ordering}

The calculation of current drive in the presence of EC resonance heating typically requires a model for the wave propagation. In the EC range of frequencies, ray-tracing techniques are routinely used as the condition of validity

$$
\lambda \ll d \ll L
$$

is usually satisfied in tokamak, where $\lambda$ is the wavelength, $d$ is the beam size and $L$ is the equilibrium non-uniformity scale length. The condition 2 assumes a well collimated beam $(\lambda \ll d)$ propagating in a plasma where $L$ is large enough such that the plasma is nearly uniform across the wave front. In a plasma, the evolution of the beam width may differ significantly from vacuum Gaussian beam theory, in which case beam-tracing methods are required. Generally, the latter must also satisfy the condition 2.

In this paper, the ray tracing code C3PO [13] is used to model the wave propagation in a fluctuating plasma, such that the typical scale length $L_{f}$ of fluctuations that can be accounted for is restricted to $L_{f} \gg d$, which sets the upper limit $p_{\max }$ to the fluctuation mode number $p$ in (1). Assuming that the condition $L_{f} \gg d$ is verified, fluctuations affect the direction of propagation but not the beam structure, which is here assumed to follow Gaussian beam theory. 


\subsection{Time scales ordering}

Resonant interaction between electrons and finite-power EC waves creates distortions of the distribution function, which in turn affect the damping rate of the wave, and may also generate a net toroidal current. In general, the evolution of the distribution function results from the competing effects of wave-particle interaction, collisions, interaction with DC electric fields, etc. It is calculated by solving the Fokker-Planck equation with the code LUKE [14]. The lifetime $\tau_{w} \simeq L / c$ of an EC wavepacket from the mirror to the damping region - is much shorter than all other relevant time scales, namely the collision time scale $\tau_{c}$ of resonant particles, the equilibrium time scale $\tau_{E}$, and the fluctuation correlation time $\tau_{f}$. Therefore, it is natural to consider an instantaneous response of the EC beam propagation and damping to changes in the fluctuating equilibrium or distribution function. A consequence of the $\tau_{w} \ll \tau_{c}$ ordering is that the self-consistency between the wave damping rate and the distribution function must be satisfied at any given time.

On the other hand, the characteristic evolution time $\tau_{c}$ of the distribution function may be of the order of or longer than the fluctuation correlation time $\tau_{f}$. The distribution function may have a "memory" of previous states in the fluctuating equilibrium. Thus, when $\tau_{f} \lesssim \tau_{c}$, a correct statistical description of the effect of fluctuations on the distribution function cannot be obtained by calculating the time-asymptotic solution to the Fokker-Planck equation for a series of independent fluctuating equilibrium states. Instead, the fluctuating equilibrium and the distribution function must evolve in a coordinated way.

An important consequence of the $\tau_{f} \lesssim \tau_{c}$ ordering is that the problem of ECCD in a fluctuating plasma requires the use of a proper solver for the time-dependent Fokker-Planck equation. The evolution time step $\tau$ for the Fokker-Planck equation is chosen such that $\tau_{f} \lesssim \tau$. With this condition, the fluctuating equilibrium states (1) at two consecutive times are decorrelated and can thus be created randomly.

\subsection{Evolution of power deposition and current profiles}

The EC power deposition profiles depends mostly upon the wave propagation path and thus fluctuates at the time scale $\tau_{f}$. The current driven by fast electrons is a moment of the distribution function and thus evolves at a time scale $\tau_{c}$. This is illustrated in Fig. 1 where two consecutive states at $t=68.4 \tau_{\text {ref }}$ and $t+\tau=69.3 \tau_{\text {ref }}$ are compared ${ }^{1}$. The power deposition profile (c) displays an immediate response to the fluctuation of the wave propagation path $(a, b)$, while the current profile keeps a memory of previous states $(d)$. A comparison with the results obtained in the absence of fluctuations shows that the width of the instantaneous power profile remains unchanged while the current profile is broadened.

The total current profile typically evolves on a much longer time scale, the resistive time scale $\tau_{R} \gg \tau_{c}$. Indeed, on a time scale $\tau_{c}$ the toroidal current profile is frozen by the large energy stored in the poloidal magnetic field such that any change in the fast electron current is instantaneously compensated by a local electric field. As a consequence, while the effect of fluctuations on the power deposition profile is a statistical scattering of the deposition location without much variation of the instantaneous deposition width, the effect on the current profile is a broadening that can be obtained from a statistical average.

\section{Effect of density fluctuations in TCV}

Fully non-inductive steady-state plasmas driven by ECCD have been achieved in the TCV tokamak $[1,4,16]$. Conventional ray-tracing/Fokker-Planck simulations systematically predict a driven current that is more than twice the experimental current (obtained from $I_{p}$ measurements using estimates of the bootstrap fraction) [3-5]. Strong evidence of fast electrons anomalous radial transport has been observed in the case of localized ECCD [17,18]. The introduction of a large level of ad-hoc radial transport in Fokker-Planck simulations of fully non-inductive discharges can reduce the calculated

${ }^{1}$ In LUKE, the reference time $\tau_{\text {ref }}$ is the thermal electron collision time for the central temperature 


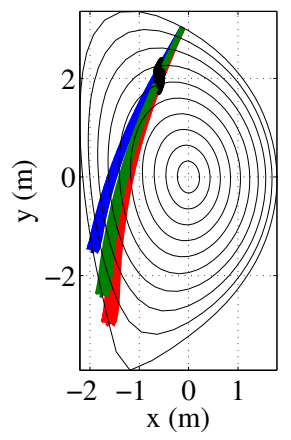

(a)

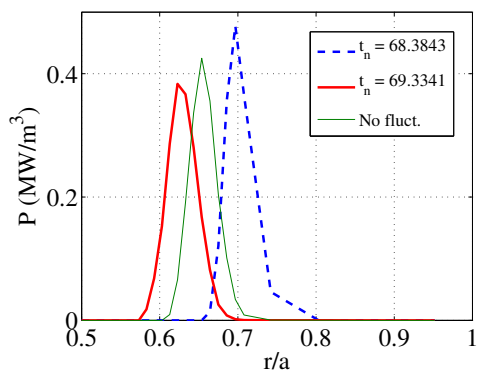

(c)

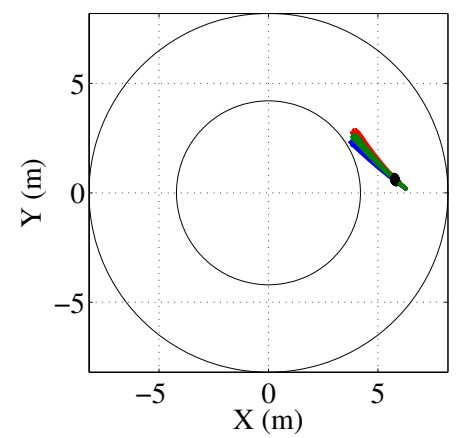

(b)

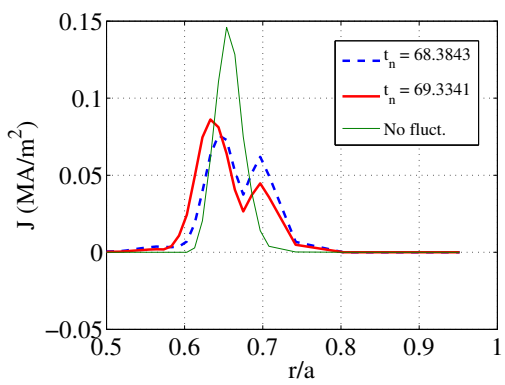

(d)

Fig. 1. EC wave propagation in the poloidal (a) and toroidal (b) planes, power deposition profile (c) and current profile (d), calculated at two successive times in a fluctuating equilibrium. The case without fluctuations is also shown. The parameters are those of Section 5.

current drive to the experimentally measured value. However, for such values of fast electrons radial transport, the fast-electron bremsstrahlung emission reconstructed from simulations is far below experimental measurements of the hard X-ray signal $[4,5]$.

Density fluctuations near the edge $(\Delta=0.02)$ are introduced in the modeling of TCV shot \#18532 in the flat-top regime $(t=1.5 \mathrm{~s})$ [5] and the results are shown in Fig. 2. The results obtained with $\sigma_{f}=$ 0.2 and $\sigma_{f}=0.6$ are fairly similar. A strong decrease of the driven current is observed (b) along with a broadening of the current profile (c). The reconstructed bremsstrahlung count rate profile as a function of chord number is quantitatively remarkably close to experimental measurements (d). With $\sigma_{f}=0.2$, the current decreases by a factor 1.5 while the count rate for the central chord decreases by a factor 2.5. With a radial diffusion coefficient of $D_{r}=0.4 \mathrm{~m}^{2} / \mathrm{s}$, the current also decreases by a factor 1.5 , but the count rate drops by a factor 8 , which is far below experimental measurements [5]. Thus, the effect of fluctuations differs significantly from that of fast electron radial transport, which is not surprising as the underlying mechanisms are very different. With density fluctuations, fast electrons are generated at radial positions that vary rapidly with time when compared to the characteristic collision time. With radial transport, fast electrons are generated at fixed radial positions and then diffuse radially while slowing down at the same time.

\section{Effect of density fluctuations in ITER}

The development of NTMs is a strong limit on performance in ITER [19], and their stabilization by ECCD is the justification for including a $20 \mathrm{MW}$ top-launch EC system in ITER [2]. Stabilization of $3 / 2$ NTMs has been successfully achieved in present day tokamaks without much apparent detrimental effect of density fluctuations [20]. However, first-harmonic O-mode ECCD in ITER could be particularly sensitive to edge density fluctuation. As the wave frequency is not far above the pedestal plasma frequency, the beam direction can be altered significantly by edge density fluctuations. In addition, the 


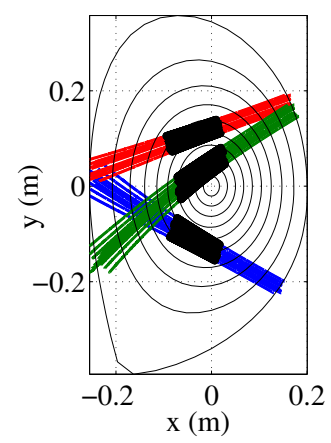

(a)

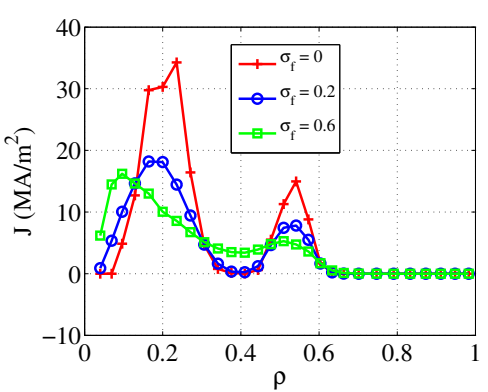

(c)

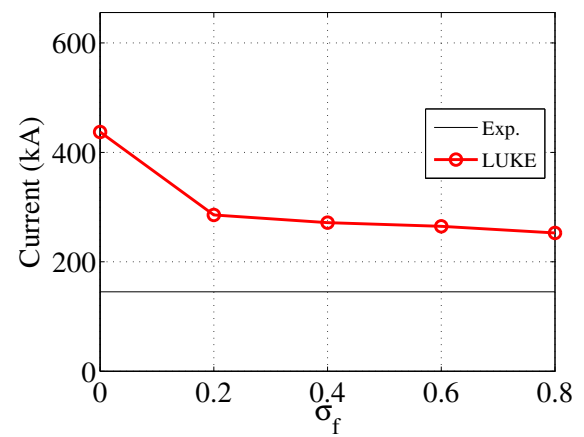

(b)

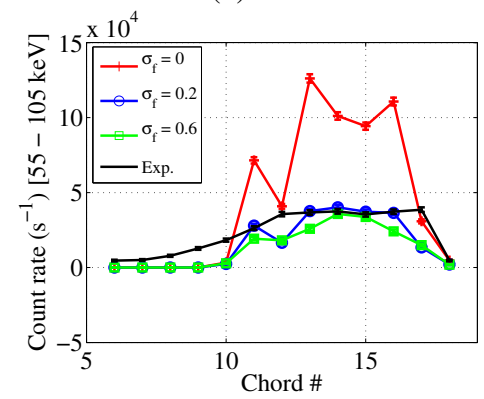

(d)

Fig. 2. EC wave propagation in unperturbed equilibrium (a); driven current (b), current profile (c), and bremsstrahlung count rate versus chord number (d) calculated for different values of $\sigma_{f}$.

relatively long propagation path means that even small variations of the edge beam direction can lead to large differences in the deposition profile.

ECCD is calculated for 3/2 NTM stabilization in the inductive ITER scenario $2[21,22]$. Results obtained in the presence of edge density fluctuations $(\Delta=0.02)$ are shown in Fig. 3 . The driven current remains unchanged (a), which is not surprising as quasilinear effects are weak for off-axis ECCD in ITER. However, the maximum current density decreases (b) as the width of the current profile (c) increases almost linearly with $\sigma_{f}$. For $\sigma_{f}=0.4$, the width of the current profile is larger than the saturated island width [2] and reaches about twice the value calculated in the absence of fluctuations (d). The broadening of the current profile is the cumulative consequence of the fluctuating power deposition profile, since it follows the standard deviation $\sigma_{p}$ of the location of power deposition (c). Both the broadening of the current profile and the fluctuating power deposition could affect the stabilization of NTMs.

\section{Conclusions}

The propagation and damping of EC waves in a fluctuating plasma equilibrium are calculated by time-dependent ray-tracing and Fokker-Planck simulations, properly accounting for the ordering of the different time scales at play : the wavepacket time is much shorter than the decorrelation time of density fluctuations, which is typically smaller or of the order of the slowing-down time of resonant electrons. All these time scales are much shorter than the equilibrium or resistive times. The resulting effect of density fluctuations is a scattering of the EC power deposition and a corresponding broadening of the current profile. In the simulations of fully non-inductive TCV discharges, density fluctuations can account for experimental observations of driven current and fast electron bremsstrahlung emission. In ITER, density fluctuation can make the stabilization of NTMs more challenging.

This work, supported by the European Communities under the contract of Association between EURATOM and CEA, was carried out within the framework of the EFDA. The views and opinions expressed herein do not necessarily reflect those of the European Commission. 


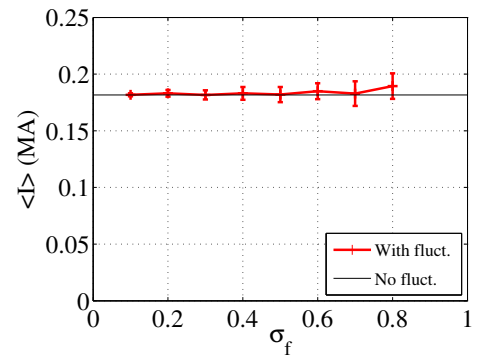

(a)

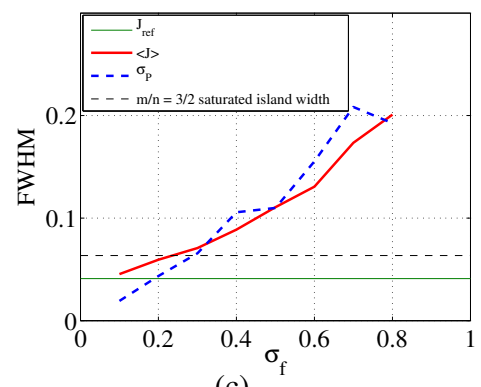

(c)

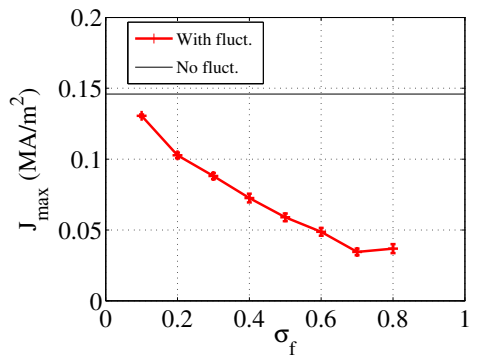

(b)

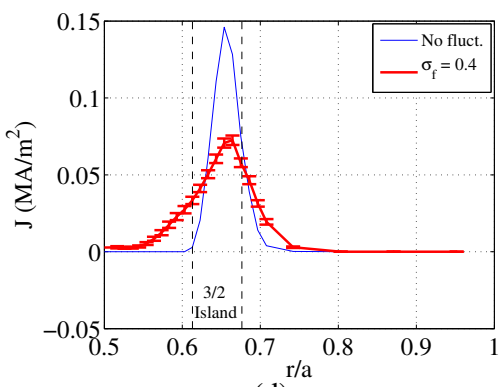

(d)

Fig. 3. Driven current (a), maximum current density (b), full width of the current profile at half maximum, and standard deviation of the power deposition profile (c) calculated for different values of $\sigma_{f}$. The current profile calculated with $\sigma_{f}=0.4$ is compared to the current profile calculated in the absence of fluctuation (d). The location $(r=1.27 \mathrm{~m})$ and width $(w=0.125 \mathrm{~m})$ of the saturated $\mathrm{m} / \mathrm{n}=3 / 2$ island [2] are shown in graphs (c) and (d).

\section{References}

1. S. Coda, et al., Plasma Phys. Control. Fusion 42 B311 (2000)

2. R. J. La Haye, et al., Nucl. Fusion, 46451 (2006)

3. R.W. Harvey, et al., Phys. Rev. Lett. 88, 205001 (2002)

4. P. Nikkola, et al., Nucl. Fusion 43, 1343 (2003)

5. J. Decker, et al., AIP Conf. Proc. 1406447 (2011)

6. E. Ott, et al., Phys. Fluids 221732 (1979)

7. P.T. Bonoli, et al., Phys. Fluids 25359 (1982)

8. G. Vahala, et al., Phys. Fluids B 44033 (1992)

9. C. Tsironis, et al., Phys. Plasmas 16112510 (2009)

10. K. Hizanidis, et al., Phys. Plasmas 171 (2010)

11. N. Bertelli, et al., J. Phys. Conf. Ser. 260012002 (2010)

12. Y. Peysson, J. Decker, L. Morini, and S. Coda, Plasma Phys. Control. Fusion 53124028 (2011)

13. Y. Peysson, J. Decker, and L. Morini, Plasma Phys. Control. Fusion 54045003 (2012)

14. J. Decker and Y. Peysson, Rep. EUR-CEA-FC-1736 (2004)

15. Y. Peysson and J. Decker, Phys. Plasmas 15, 092509 (2008)

16. O. Sauter, et al., Phys. Rev. Lett. 84, 3322 (2000)

17. S. Coda, et al., Nucl. Fusion 43, 1361 (2003)

18. S. Coda, et al., Plasma Phys. Control. Fusion 48, B359 (2006)

19. T.C. Hender, et al., Nucl. Fusion 47 S128 (2007)

20. R. J. La Haye, et al., Phys. Plasmas, 13055501 (2006)

21. Y. Gribov, Available online: ftp.jp.iter.org (2006)

22. R. Prater, et al., Nucl. Fusion 48035006 (2008) 